\title{
PRODUCTS OF QUASI-COMPLEXES
}

THOMAS R. BRAHANA

It is the purpose of this note to show that the product space of two quasi-complexes is a quasi-complex. Quasi-complexes are spaces defined by Lefschetz in connection with the theory of fixed points. A product of quasi-complexes has the fixed point property then, if the Lefschetz number is not zero. This question was pointed out by Eldon Dyer at the Institute for Point Set Topology in Madison $[5$, p. 88].

1. Definitions. The following definition, due to Lefschetz, is in [4, p. 322].

Let $X$ be a compact space, $\left\{U_{\alpha}\right\}$ a set of open coverings of $X$, cofinal in the set of all open coverings, indexed by $\{\alpha\}$. Let $N\left(U_{\alpha}\right)$ be the nerve of the covering $U_{\alpha}$, and if $\alpha<\beta$, let $\pi_{\alpha \beta}: N\left(U_{\beta}\right) \rightarrow N\left(U_{\alpha}\right)$ be one of the projections induced by the inclusion relations associated with the refinement of $U_{\alpha}$ by $U_{\beta}$. The set of all projections is denoted by $\left\{\pi_{\alpha \beta}\right\}$.

Further for each $\alpha \in\{\alpha\}$, there exists a $\beta \in\{\alpha\}, \alpha<\beta$, and one or more chain mappings $\omega_{\beta \alpha}: N\left(U_{\alpha}\right) \rightarrow N\left(U_{\beta}\right)$, called antiprojections, such that

(a) $\omega_{\beta \alpha} \pi_{\alpha \beta} \sim 1$,

(b) if $\alpha<\beta<\gamma$, and if $\omega_{\gamma \beta}, \omega_{\beta \alpha}$, are anti-projections, so is $\omega_{\gamma \beta} \omega_{\beta \alpha}$,

(c) if $\omega_{\beta \alpha}$ and $\omega_{\beta \alpha}^{\prime}$ are anti-projections, then $\omega_{\beta \alpha} \sim \omega_{\beta \alpha}^{\prime}$.

If $\sigma_{\gamma}$ is a simplex of $N\left(U_{\gamma}\right)$, let $\left[\sigma_{\gamma}\right]$ denote the kernel of $\sigma_{\gamma}$, that is, the intersection of the sets of $U_{\gamma}$ corresponding to vertices of $\sigma_{\gamma}$. Further, if $c^{p}$ is a chain of $N\left(U_{\gamma}\right)$, let $\left[c^{p}\right]$ denote the union of the kernels of simplexes in the carrier of $c^{p}$.

(d) all indices being understood in $\{\alpha\}$, for every $\alpha$ there is a $\gamma, \gamma>\alpha$, and for every $\beta$ an $\eta>\beta, \gamma$ (depending on $\alpha, \beta$ ) such that $\omega_{\eta \gamma}$ exists, satisfies (a), (b), (c), and if the simplex $\sigma_{\gamma} \in N\left(U_{\gamma}\right)$, then $\left\{\left[\sigma_{\gamma}\right] \cup\left[\omega_{\eta \gamma} \sigma_{\gamma}\right]\right\}$ is contained in a set of $U_{\alpha}$.

The collection $\left(X ;\left\{U_{\alpha}\right\} ;\left\{\pi_{\alpha \beta}\right\} ;\left\{\omega_{\beta \alpha}\right\}\right)$ defines a quasi-complex $X$.

2. Products of quasi-complexes. Let $\left(X ;\left\{U_{\alpha}\right\} ;\left\{\pi_{\alpha \beta}\right\} ;\left\{\omega_{\beta \alpha}\right\}\right)$ and $\left(Y ;\left\{V_{\alpha^{\prime}}\right\} ;\left\{\bar{\pi}_{\alpha^{\prime} \beta^{\prime}}\right\} ;\left\{\bar{\omega}_{\beta^{\prime} \alpha^{\prime}}\right\}\right)$ be two quasi-complexes. In order to show that $X \times Y$ is a quasi-complex it is sufficient to show that the 1955.

Presented to the Society, November 18, 1955; received by the editors October 24, 
existence of projections and anti-projections on the nerves of coverings of $X$ and $Y$ insures the existence of projections and anti-projections on the nerves of the product coverings of $X \times Y$, and that these mappings satisfy the conditions (a) $-(d)$.

The nerve of the product covering $\left\{U_{\alpha} \times V_{\alpha^{\prime}}\right\}$ is given by the simplicial product $N\left(U_{\alpha}\right) \Delta N\left(V_{\alpha^{\prime}}\right)$ of the nerves, $[2$, p. $66 ; 3$, p. 204], the notation used here being as follows. Let $u_{0}, u_{1}, \cdots$, be the vertices of $N\left(U_{\alpha}\right), v_{0}, v_{1}, \cdots$ be the vertices of $N\left(V_{\alpha^{\prime}}\right)$, and $\left(u_{0}, v_{0}\right)$, $\left(u_{0}, v_{1}\right), \cdots$ the vertices of $N\left(U_{\alpha}\right) \triangle N\left(V_{\alpha^{\prime}}\right)$. Let the image under the projection $\pi_{\alpha \beta}$ of the vertex $u_{0}$ be $\pi_{\alpha \beta} u_{0}$, the image under $\bar{\pi}_{\alpha^{\prime} \beta^{\prime}}$ of the vertex $v_{0}$ be $\bar{\pi}_{\alpha^{\prime} \beta}, v_{0}$.

Associated with the projections $\pi_{\alpha \beta}$ and $\bar{\pi}_{\alpha^{\prime} \beta^{\prime}}$ is the map $\pi_{\alpha \beta} \Delta \bar{\pi}_{\alpha^{\prime} \beta^{\prime}}$ : $N\left(U_{\beta}\right) \Delta N\left(V_{\beta^{\prime}}\right) \rightarrow N\left(U_{\alpha}\right) \Delta N\left(V_{\alpha^{\prime}}\right)$, defined for simplexes as $\pi_{\alpha \beta}$ $\Delta \bar{\pi}_{\alpha^{\prime} \beta^{\prime}}:\left(\left(u_{0}, v_{0}\right)\left(u_{1}, v_{1}\right) \cdots\right) \rightarrow\left(\left(\pi_{\alpha \beta} u_{0}, \bar{\pi}_{\alpha^{\prime} \beta^{\prime}} v_{0}\right)\left(\pi_{\alpha \beta} u_{1}, \bar{\pi}_{\alpha^{\prime} \beta^{\prime}, v_{1}}\right) \cdots\right)$. This is a projection associated with the inclusion of the sets of the product coverings, and in this manner $X \times Y$ is provided with the coverings, nerves and projections of the definition.

Using the existence of anti-projections $\omega_{\beta \alpha}$ and $\bar{\omega}_{\beta^{\prime} \alpha^{\prime}}$, a mapping $\omega_{\beta \alpha} \Delta \bar{\omega}_{\beta^{\prime} \alpha^{\prime}}: N\left(U_{\alpha}\right) \Delta N\left(V_{\alpha^{\prime}}\right) \rightarrow N\left(U_{B}\right) \Delta N\left(V_{\beta^{\prime}}\right)$ will be constructed.

Denote by $N\left(U_{\alpha}\right) \times N\left(V_{\alpha^{\prime}}\right)$ the cartesian product of $N\left(U_{\alpha}\right)$ and $N\left(V_{\alpha^{\prime}}\right)\left[2\right.$, p. 67]. Each simplex of $N\left(U_{\alpha}\right) \times N\left(V_{\alpha^{\prime}}\right)$ is in some complex of the form $\sigma^{p} \times \tau^{q}$; denote the geometric realization of this complex by $\left|\sigma^{p} \times \tau^{q}\right|$. Let the vertices $u_{0}, u_{1}, \ldots$ of $N\left(U_{\alpha}\right)$ and $v_{0}, v_{1}, \cdots$ of $N\left(V_{\alpha^{\prime}}\right)$ be ordered by $u_{0}<u_{1}<\cdots$ and $v_{0}<v_{1}<\cdots$. Suppose $\sigma^{p}=\left(u_{0} u_{1} \cdots u_{p}\right), \tau^{q}=\left(v_{0} v_{1} \cdots v_{q}\right)$, then a $p+q$-simplex $A=\left(\left(u_{0}, v_{0}\right)\right.$ $\left.\cdot\left(u_{0}, v_{1}\right) \cdots\left(u_{0}, v_{q}\right)\left(u_{1}, v_{q}\right) \cdots\left(u_{p}, v_{q}\right)\right)$, is uniquely determined, the first $q+1$ vertices having the same $u$-coordinate, the last $p+1$ vertices having the same $v$-coordinate. Also the $p+q-1$-simplex $\hat{A}=\left(\left(u_{0}, v_{0}\right)\right.$ $\left.\cdot\left(u_{0}, v_{1}\right) \cdots\left(u_{0}, v_{q-1}\right)\left(u_{1}, v_{q}\right) \cdots\left(u_{p}, v_{q}\right)\right)$ is uniquely determined.

A retraction $\bar{\eta}^{p+q}:\left|\sigma^{p} \times \tau^{q}\right| \rightarrow\left|\sigma^{p} \times \tau^{q}\right|$ will be constructed with the properties

(1) the boundary of $\left|\sigma^{p} \times \tau^{q}\right|$ is mapped onto itself,

(2) the simplex $A$ is mapped onto $\left|\sigma^{p} \times \tau^{q}\right|$,

(3) the image of the interior of each simplex is the interior of a set of the form $U\left|\sigma^{k} \times \tau^{l}\right|$,

(4) the retraction induces a simplicial map on one subdivision of $\sigma^{p} \times \tau^{q}$ to another subdivision of $\sigma^{p} \times \tau^{q}$.

For simplexes of the form $u_{i} \times \tau_{j}, \sigma_{k} \times v_{l}, u_{i}, v_{l}$ being vertices, $\bar{\eta}^{s}$ is the identity. For $\left|\sigma^{p} \times \tau^{q}\right|$ the retraction $\bar{\eta}^{p+q}$ is defined recursively with respect to dimension; let $\bar{\lambda}^{p+q}$ be the stereographic projection from $\left(u_{0}, v_{q}\right)$ of the space of $\hat{A}$ to the space of simplexes of the form $B=\left|\left(u_{0} u_{1} \cdots u_{p}\right) \times\left(v_{0} v_{1} \cdots v_{q-1}\right)\right| \cup\left|\left(u_{1} \cdots u_{p}\right) \times\left(v_{0} \cdots v_{q}\right)\right|$; 
there is a ray of the stereographic projection through each point of $\left|\sigma^{p} \times \tau^{q}\right| ; \bar{\lambda}^{p+q}$ maps each point of the ray $r$ not in the (closed) simplex $A$ into the intersection of the ray $r$ with $B$. The points of $A \cap r$ are mapped by a continuous stretching which leaves $\left(u_{0}, v_{q}\right)$ fixed, and sends $r \cap \hat{A}$ into $r \cap B$, in such a way that the map is continuous on $\left|\sigma^{p} \times \tau^{q}\right|$ into $\left|\sigma^{p} \times \tau^{q}\right|$.

Finally if in the space $B$ the maps $\bar{\eta}^{p+q-1}$ (which are defined using order relations among the vertices of $B$ ), are such that $\bar{\eta}^{p+q-1}\left(x_{1}\right)=x_{2}$ and $r_{1}$ and $r_{2}$ are the rays from $\left(u_{0}, v_{q}\right)$ to $x_{1}$ and $x_{2}$ respectively, then $\bar{\mu}^{p+q}$ is defined as the map which maps $r_{1}$ onto $r_{2}$, leaving $\left(u_{0}, v_{q}\right)$ fixed, sending $r_{1} \cap \hat{A}$ into $r_{2} \cap \hat{A}, r_{1} \cap B$ into $r_{2} \cap B$, and is continuous of $\left|\sigma^{p} \times \tau^{q}\right|$ onto $\left|\sigma^{p} \times \tau^{q}\right|$.

The map $\bar{\eta}^{p+q}$ is the composition $\bar{\mu}^{p+q} \bar{\lambda}^{p+q}$, and it can be seen that $\bar{\eta}^{p+q}$ has properties (1), (2), (3) that were prescribed.

In order to check (4), note that the inverse image of the simplicial decomposition of $B$ under $\bar{\lambda}^{p+q}$ provides $\hat{A}$ with a simplicial decomposition. $A$ is the join of $\hat{A}$ with the point $\left(u_{0}, v_{q}\right)$, and the join decomposition is defined on $A$. Also there is a decomposition for the remainder of $\left|\sigma^{p} \times \tau^{q}\right|$, whose simplexes are formed from the points of $\left(\left|\sigma^{p} \times \tau^{q}\right|-A\right) \cap($ the rays which intersect a fixed simplex of $A$ ), this set being made a geometric complex without the introduction of new vertices. The complex formed from this decomposition of $\left|\sigma^{p} \times \tau^{q}\right|$ will be denoted $K_{1}$.

The inverse image under $\bar{\lambda}^{p+q}$ of the simplicial decomposition of $\left|\sigma^{p} \times \tau^{q}\right|$, as a cartesian product, provides another simplicial decomposition of $A$. This, together with the decomposition of $K_{1}$ for the remainder of $\left|\sigma^{p} \times \tau^{q}\right|$, provides $\left|\sigma^{p} \times \tau^{q}\right|$ with a simplicial decomposition, and this complex will be denoted $K_{2}$.

The mapping induced by $\bar{\eta}^{p+q}$ of $K_{2}$ on $K_{1}$ can be seen to be simplicial. This induces chain mappings on the chain groups of $K_{2}$ to $K_{1}$. Now $\bar{\eta}^{p+q}$ induces a map of chains of $\sigma^{p} \times \tau^{q}$ into chains of $\sigma^{p} \times \tau^{q}$; i.e. if $D$ is an oriented $r$-simplex of $\sigma^{p} \times \tau^{q}$, and $\bar{\eta}^{p+q}(|D|)=U\left|\sigma^{k} \times \tau^{l}\right|$, the $r$-chain whose coefficient is +1 for $D$ and 0 for all other simplexes is mapped into the $r$-chain $\sum a_{i} D_{i}^{\prime}$, where $D_{i}^{\prime}$ are $r$-simplexes in $\sigma^{p} \times \tau^{q}$, and $a_{i}$ is 0 if $\left|D_{i}^{\prime}\right|$ is not contained in $U\left|\sigma^{k} \times \tau^{l}\right|$; otherwise the simplex $\left(\bar{\eta}^{p+q}\right)^{-1}\left|D_{i}^{\prime}\right|=\left|D_{i}\right|$ is a geometric simplex of the subdivision $K_{1}$ of $\left|\sigma^{p} \times \tau^{q}\right|$, and if $D_{i}^{\prime}$ is oriented, the condition that $\left(\bar{\eta}^{p+q}\right)^{-1}$ preserve order of vertices provides $D_{i}$ with an orientation, and if this simplex is coherently oriented as a subdivision with the orientation of $D$, then $a_{i}=+1$; if not coherently oriented, $a_{i}=-1$. Let this map be denoted $\eta$.

It can be seen that $\eta: C\left(\sigma^{p} \times \tau^{q}\right) \rightarrow C\left(\sigma^{p} \times \tau^{q}\right)$ is a chain map, i.e. 
$\eta \partial=\partial \eta$, for $\bar{\eta}^{p+q}$ induces a simplicial map of $K_{2}$ on $K_{1}$, and every simplex of $\sigma^{p} \times \tau^{q}$ corresponds under the injection map to a chain of $K_{2}$, which is mapped onto a chain of $K_{1}$ which corresponds (see above) to a chain of $\sigma^{p} \times \tau^{q}$. Since each of these maps is a chain map, the composition is.

A map $\eta$ applied to each of the principal simplexes of $N\left(U_{\alpha}\right)$ $\times N\left(V_{\alpha^{\prime}}\right)$ provides the desired map of $N\left(U_{\alpha}\right) \times N\left(V_{\alpha^{\prime}}\right)$ into $N\left(U_{\alpha}\right)$ $\times N\left(V_{\alpha^{\prime}}\right)$. These maps agree on the common faces of the principal simplexes, due to the fact that they are defined in the same way for simplexes of less than maximum dimension.

Associated with each chain corresponding to the complex of the form $\sigma^{p} \times \tau^{q}$ there is defined for each pair of antiprojections $\omega_{\beta \alpha}$ and $\bar{\omega}_{\beta^{\prime} \alpha^{\prime}}$ a map $\phi\left(\sigma^{p} \times \tau^{q}\right)=\omega_{\beta \alpha} \sigma^{p} \times \bar{\omega}_{\beta^{\prime} \alpha^{\prime}} \tau^{q}$, where if $\omega_{\beta \alpha} \sigma^{p}=a_{0} \bar{\sigma}_{0}^{p}+a_{1} \bar{\sigma}_{1}^{p}$ $+\cdots$, and $\bar{\omega}_{\beta^{\prime} \alpha^{\prime}} \tau^{q}=b_{0} \bar{\tau}_{0}^{q}+b_{1} \bar{\tau}_{1}^{q}+\cdots, \omega_{\beta \alpha} \sigma^{p} \times \bar{\omega}_{\beta^{\prime} \alpha^{\prime}} \tau^{q}=a_{0} b_{0}\left(\bar{\sigma}_{0}^{p} \times \bar{\tau}_{0}^{q}\right)$ $+a_{0} b_{1}\left(\bar{\sigma}_{0}^{p} \times \bar{\tau}_{1}^{q}\right)+\cdots$ Noting that $\partial\left(\sigma^{p} \times \tau^{q}\right)=\partial \sigma^{p} \times \tau^{q}+(-1)^{p} \sigma^{p}$ $\times \partial \tau^{q}$ it can be seen that $\partial \phi=\phi \partial$.

The map $\phi \eta: N\left(U_{\alpha}\right) \times N\left(V_{\alpha^{\prime}}\right) \rightarrow N\left(U_{\beta}\right) \times N\left(V_{\beta^{\prime}}\right)$ is defined and a chain map, since $\phi$ and $\eta$ are, and will be denoted by $\omega_{\beta \alpha} \times \bar{\omega}_{\beta^{\prime} \alpha^{\prime}}$. This map is extended to the map $\omega_{\beta \alpha} \Delta \bar{\omega}_{\beta^{\prime} \alpha^{\prime}}: N\left(U_{\alpha}\right) \Delta N\left(V_{\alpha^{\prime}}\right) \rightarrow N\left(U_{\beta}\right)$ $\Delta N\left(V_{\beta^{\prime}}\right)$ by defining $\omega_{\beta \alpha} \Delta \bar{\omega}_{\beta^{\prime} \alpha^{\prime}}=\left(\omega_{\beta \alpha} \times \bar{\omega}_{\beta^{\prime} \alpha^{\prime}}\right) \theta$, where $\theta$ is the chain mapping associated with the deformation retraction of $\mid N\left(U_{\alpha}\right)$ $\triangle N\left(V_{\boldsymbol{\alpha}^{\prime}}\right) \mid$ into $\left|N\left(U_{\boldsymbol{\alpha}}\right) \times N\left(V_{\boldsymbol{\alpha}^{\prime}}\right)\right|$, which is given in [2, p. 69]. Clearly $\omega_{\beta \alpha} \Delta \bar{\omega}_{\beta^{\prime} \alpha^{\prime}}$ is a chain map, and it remains to check the conditions (a)-(d) of the definition of a quasi-complex.

Denote the tensor product of the complexes $N\left(U_{\boldsymbol{\alpha}}\right)$ and $N\left(V_{\boldsymbol{\alpha}^{\prime}}\right)$ by $N\left(U_{\alpha}\right) \otimes N\left(V_{\alpha^{\prime}}\right)[3$, p. 201].

To check (a), the diagram

$$
\begin{aligned}
& N\left(U_{\boldsymbol{\alpha}}\right) \Delta N\left(V_{\boldsymbol{\alpha}^{\prime}}\right) \stackrel{\theta}{\stackrel{\theta}{\rightleftarrows}} N\left(U_{\boldsymbol{\alpha}}\right) \times N\left(V_{\boldsymbol{\alpha}^{\prime}}\right) \stackrel{\stackrel{\zeta}{\rightleftarrows}}{\leftarrow} N\left(U_{\boldsymbol{\alpha}}\right) \otimes N\left(V_{\boldsymbol{\alpha}^{\prime}}\right) \\
& \uparrow \pi_{\alpha \beta} \Delta \bar{\pi}_{\alpha^{\prime} \beta^{\prime}} \downarrow \omega_{\beta \alpha} \Delta \bar{\omega}_{\beta^{\prime} \alpha^{\prime}} \quad \uparrow \pi_{\alpha \beta} \otimes \bar{\pi}_{\alpha^{\prime} \beta^{\prime}} \downarrow \omega_{\beta \alpha} \otimes \bar{\omega}_{\beta^{\prime} \alpha^{\prime}} \\
& N\left(U_{\beta}\right) \Delta N\left(V_{\beta^{\prime}}\right) \stackrel{\stackrel{\theta^{\prime}}{\rightleftarrows}}{\stackrel{\theta^{\prime}}{\leftarrow}} N\left(U_{\beta}\right) \times N\left(V_{\beta^{\prime}}\right) \stackrel{\stackrel{\zeta^{\prime}}{\rightleftarrows}}{\leftrightarrows} N\left(U_{\beta}\right) \otimes N\left(V_{\beta^{\prime}}\right)
\end{aligned}
$$

where $\theta, \tilde{\theta}, \theta^{\prime}, \tilde{\theta}^{\prime}$, are the maps induced on the chain groups by the deformation retractions, $\zeta, \tilde{\zeta}, \zeta^{\prime}, \tilde{\zeta}^{\prime}$, are maps as defined in [3], has the properties $\left(\pi_{\alpha \beta} \Delta \bar{\pi}_{\alpha^{\prime} \beta^{\prime}}\right) \sim \tilde{\theta} \tilde{\zeta}\left(\pi_{\alpha \beta} \otimes \bar{\pi}_{\alpha^{\prime} \beta^{\prime}}\right) \zeta^{\prime} \theta^{\prime},\left(\omega_{\beta \alpha} \Delta \bar{\omega}_{\beta^{\prime} \alpha^{\prime}}\right) \sim \tilde{\theta}^{\prime} \tilde{\zeta}^{\prime}\left(\omega_{\beta \alpha}\right.$ $\left.\otimes \bar{\omega}_{\beta^{\prime} \alpha^{\prime}}\right) \zeta \theta,\left(\omega_{\beta \alpha} \otimes \bar{\omega}_{\beta^{\prime} \alpha^{\prime}}\right)\left(\pi_{\alpha \beta} \otimes \bar{\pi}_{\alpha^{\prime} \beta^{\prime}}\right)=\omega_{\beta \alpha} \pi_{\alpha \beta} \otimes \bar{\omega}_{\beta^{\prime} \alpha^{\prime}} \bar{\pi}_{\alpha^{\prime} \beta^{\prime}} \sim 1$, from which it is deduced that $\left(\omega_{\beta \alpha} \Delta \bar{\omega}_{\beta^{\prime} \alpha^{\prime}}\right)\left(\pi_{\alpha \beta} \Delta \bar{\pi}_{\alpha^{\prime} \beta^{\prime}}\right) \sim 1$. This is precisely condition (a). Condition (b) is immediate, and condition (c) is checked in the same manner as (a). 
To establish condition (d), it is first shown that for normal spaces, (d) is equivalent to $\left(d^{\prime}\right)$ below.

Denote the star of $\sigma_{\gamma}$ (i.e. the union of all sets which correspond to vertices of $\left.\sigma_{\gamma}\right)$ by $\left\langle\sigma_{\gamma}\right\rangle$; define $\left|\omega_{\eta \gamma} C\left(\sigma_{\gamma}\right)\right|$ to be the union of the stars of simplexes with nonzero coefficients in the images under $\omega_{\eta \gamma}$ of chains of the complex formed by $\sigma_{\gamma}$ and its faces.

Condition ( $\left.\mathrm{d}^{\prime}\right)$ : For every $\alpha$ there exists a $\gamma, \gamma>\alpha$, and for every $\beta$ an $\eta(\alpha, \beta), \eta>\gamma, \beta$, and a mapping $\omega_{\eta \gamma}$ such that $\left\{\left\langle\sigma_{\gamma}\right\rangle \cup\left|\omega_{\eta \gamma} C\left(\sigma_{\gamma}\right)\right|\right\}$ $>U_{\alpha}$.

Since in a normal space every finite open covering $U_{\alpha}$ has a finite star-refinement $U_{\alpha_{1}}$, condition (d) may be applied to $\alpha_{1}, \gamma, \beta, \eta$. Since $\left[\sigma_{\gamma}\right]>U_{\alpha_{1}},\left\langle\sigma_{\gamma}\right\rangle$ is contained in the star of some set of $U_{\alpha_{1}}$. Also, if $\sigma_{\gamma}^{i}$ is any face of $\sigma_{\gamma}$, since $\left[\sigma_{\gamma}^{i}\right] \cup\left[\omega_{\eta \gamma} \sigma_{\gamma}^{i}\right]$ is in some set of $U_{\alpha_{1}}$ which intersects the set which contains $\left\langle\sigma_{\gamma}\right\rangle$ (i.e. $\left[\sigma_{\gamma}^{i}\right] \cap\left\langle\sigma_{\gamma}\right\rangle \neq 0$ ) it follows that $\left\{\left\langle\sigma_{\gamma}\right\rangle \cup\left|\omega_{\eta \gamma} C\left(\sigma_{\gamma}\right)\right|\right\} \subset$ star of some set of $U_{\alpha_{1}}$, hence $\left\{\left\langle\sigma_{\gamma}\right\rangle\right.$ $\left.\cup\left|\omega_{\text {vr }} C\left(\sigma_{\gamma}\right)\right|\right\}>U_{\alpha}$.

Thus using mappings $\omega_{\eta \gamma}$ and $\bar{\omega}_{\eta^{\prime} \gamma^{\prime}}$ satisfying (d'), the mapping $\omega_{\eta \gamma} \Delta \bar{\omega}_{\eta^{\prime} \gamma^{\prime}}$ satisfies $\left(d^{\prime}\right)$, since

$$
\left|\left(\omega_{\eta \gamma} \Delta \bar{\omega}_{\gamma^{\prime} \gamma^{\prime}}\right) C\left(\sigma_{\gamma} \Delta \tau_{\gamma^{\prime}}\right)\right| C\left|\omega_{\eta \gamma} C\left(\sigma_{\gamma}\right)\right| \times\left|\bar{\omega}_{\eta^{\prime} \gamma^{\prime}} C\left(\tau_{\gamma^{\prime}}\right)\right| .
$$

This completes the check, and the following may be stated.

Theorem. The product of two quasi-complexes is a quasi-complex.

\section{REFERENCES}

1. E. Dyer, $A$ fixed point theorem, Proc. Amer. Math. Soc. vol. 7 (1956) pp. 662672.

2. S. Eilenberg and N. Steenrod, Foundations of algebraic topology, Princeton, 1952.

3. S. Eilenberg and J. A. Zilber, On products of spaces, Amer. J. Math. vol. 75 (1953) pp. 200-204.

4. S. Lefschetz, Algebraic topology, Amer. Math. Soc. Colloquium Publications, vol. 27, 1942.

5. Summary of lectures and seminars, Summer Institute on Set Theoretic Topology, Madison, Wisconsin, 1955.

UNIVERSITY OF GEORGIA 